\title{
Gestión de calidad del servicio y la satisfacción del cliente en los restaurantes de Sullana
}

\section{Management of Service Quality and Customer Satisfaction in Restaurants, Sullana}

\author{
Carlos David Ramos Rosas ${ }^{1}$ https://orcid.org/0000-0002-5868-2441 \\ ${ }^{1}$ Universidad Católica Los Ángeles de Chimbote, Sullana, Perú \\ cramosrauladech.edu.pe
}

\section{(c) $(1)(8$}

Esta obra está bajo una licencia internacional

Creative Commons Atribución-NoComercial 4.0.

$\begin{array}{ll}\text { Enviado: } & 2020 / 09 / 30 \\ \text { Aceptado: } & 2021 / 09 / 14 \\ \text { Publicado: } & 2021 / 12 / 30\end{array}$

\section{Resumen}

La calidad del servicio y la satisfacción del cliente son variables que las empresas MYPE deben tener en cuenta para alcanzar un alto nivel de competitividad. El presente artículo se planteó como objetivo general determinar la relación entre la calidad del servicio y la satisfacción del cliente en los restaurantes de Sullana (Perú) durante el 2019. El diseño y el enfoque de la investigación corresponden al tipo de investigación cuantitativa-no experimental $\mathrm{y}$ descriptiva-correlativa. De una población infinita de clientes que acuden a los restaurantes ubicados en el distrito de Sullana, se tomó una muestra de 384 clientes, a quienes se les aplicó una encuesta cuyos resultados se presentan a continuación. En relación con la calidad del servicio, el $68.3 \%$ considera estar totalmente satisfecho. Con respecto a la satisfacción de los clientes, el $69 \%$ está totalmente satisfecho; el $45.8 \%$ concuerda en que los establecimientos del distrito cuentan con equipos modernos, instalaciones y materiales atractivos, así como con personal de apariencia pulcra; el $41.1 \%$ está totalmente satisfecho con la capacidad de respuesta; por otro lado, el $57.8 \%$ de los clientes está satisfecho con el servicio de seguridad brindado dentro de las instalaciones. Finalmente, se concluye que la calidad del servicio se relaciona significativamente con la satisfacción del cliente en los restaurantes de Sullana.

Palabras clave: calidad, servicio, restaurante, satisfacción, cliente, MYPE.

Sumario: Introducción, Metodología, Resultados, Discusión y Conclusiones.

Como citar: Ramos, C. (2021). Gestión de calidad del servicio y la satisfacción del cliente en los restaurantes de Sullana. Revista Tecnológica - Espol, 33(3), 230-241.

http://www.rte.espol.edu.ec/index.php/tecnologica/article/view/773 


\begin{abstract}
Service quality and customer satisfaction are variables that SMEs (Small and Medium-Sized enterprises, acronyms in English) should take into account to achieve a high level of competitiveness. The general objective of this article was to determine the relationship between service quality and customer satisfaction in restaurants in Sullana (Peru) during 2019. The design and approach of this research were quantitative-non-experimental and descriptivecorrelative research. From an infinite population of customers who go to restaurants located in the Sullana district, a sample of 384 customers was taken to apply the survey. Results are presented as follows: regarding the service quality, $68.3 \%$ consider themselves to be fully satisfied. Concerning customer satisfaction, $69 \%$ are fully satisfied while $45.8 \%$ agree that the establishments in the district have modern equipment, attractive facilities, and materials, as well as neat looking staff. Also, $41.1 \%$ are fully satisfied with the response capacity, and $57.8 \%$ of the surveyed customers are satisfied with the security service provided within the premises. Finally, it is concluded that service quality is significantly related to customer satisfaction in Sullana restaurants.
\end{abstract}

Keywords: Quality, Service, Restaurants, Satisfaction, Customer, SME.

\title{
Introducción
}

Sullana destaca como un distrito gastronómico desde tiempos inmemoriales, ya que la variedad de platos que ofrecen sus restaurantes proviene de recetas trasmitidas de generación en generación. Por otro lado, este acervo culinario cobra relevancia y se hace más sofisticado gracias al aporte que realizan las carreras e institutos de gastronomía locales y regionales.

La diversidad cultural que existe en el Perú se puede comprobar en la variedad de platos que se preparan en los distintos restaurantes asentados a lo largo del país. Por ejemplo, Rodríguez \& Venturo (2007) han hecho un registro de 491 platos típicos a nivel nacional.

Además, cabe señalar que la gastronomía en el Perú sigue en crecimiento; datos oficiales afirman que los servicios de restaurantes se incrementaron en un $3.01 \%$ durante el año 2018. Este crecimiento incluye restaurantes turísticos, comidas rápidas, pollerías, comida criolla y cevicheras, en los que se evidencian la presencia de atención personalizada, nuevas propuestas gastronómicas, promociones y descuentos (INEI, 2018).

Los restaurantes de nivel internacional tienen un alto estándar de calidad. "Al igual que los hoteles, los restaurantes también reciben una clasificación basada en varios conceptos: Instalaciones, servicios, menú, etc., siendo el servicio de los camareros en las mesas uno de los criterios más valorados" (Gastromakers, 2018).

La prioridad de todo establecimiento es garantizar la salubridad en el expendio de alimentos. En este caso, el sistema de gestión alimentaria es algo indefectible en el procesamiento de los alimentos. Para ello, se cuenta con la NORMA ISO 22001 como herramienta imprescindible para garantizar la seguridad alimentaria. Esta norma tiene la función de reglamentar los procedimientos que se deben seguir y certificar el cumplimiento de estos. Hay que tener presente que, para tener prestigio y mejorar la imagen frente a los clientes, es necesario que los establecimientos incorporen normativas de calidad, como, por ejemplo, la $Q$ de la Calidad Turística (Jiménez, 2016).

J. Coronel et al. (2019) plantearon, como objetivo general de su estudio, determinar la calidad del servicio en restaurantes de mariscos de la ciudad de Riobamba, Ecuador. Los resultados mostraron que existe una brecha negativa en las cinco dimensiones de la calidad del 
servicio evaluadas (tangibles, confiabilidad, respuesta, seguridad y empatía); es decir, no existe correlación entre las variables analizadas. En consecuencia, el conjunto de los administradores de los restaurantes debería reformar el entorno físico y proporcionar instalaciones actualizadas y suficientes para la demanda, así como prestar especial atención a las variables analizadas.

Por su lado, Vera \& Trujillo (2017) redactaron en español una escala de medición para valorar la calidad en el servicio de los restaurantes que ofrecen atención a la mesa, denominada "Escala Mexicana de Calidad en el Servicio en Restaurantes" (EMCASER). Un aporte importante de esta escala radica en que los parámetros de su medición recogen un conjunto amplio de atributos de este sector, en lugar de partir de las dimensiones genéricas del instrumento de medición de calidad en el servicio más citado en la literatura: Servqual.

Pintado Alberca (2018) planteó como objetivo general de su investigación determinar y describir la caracterización de la calidad del servicio al cliente y la rentabilidad de las MYPE. Su estudio concluyó que los servicios brindados son buenos, ya que emplean adecuadamente elementos tangibles, como los materiales de comunicación para la oferta de sus productos. Asimismo, se observó una adecuada capacidad de los negocios para resolver las dudas e inquietudes de sus clientes, así como profesionalismo, cortesía y una buena capacidad de respuesta en la atención y entrega de pedidos, respetando los tiempos y precios ofrecidos.

Por su parte, en su estudio, López (2018) se propuso determinar la relación de la calidad del servicio y la satisfacción de los clientes del restaurante Rachy's de la ciudad de Guayaquil. Los resultados de esta investigación identificaron la insatisfacción de los clientes respecto a las dimensiones de respuesta inmediata y empatía. Por otro lado, se pudo evidenciar que existe una correlación positiva entre la calidad del servicio y la satisfacción al cliente.

En su investigación, C. Coronel (2016) se planteó como objetivo determinar la calidad del servicio y el grado de satisfacción del cliente en el restaurant Pizza Hut del centro comercial Mega Plaza Lima. El estudio arrojó como resultado que $41.6 \%$ de los clientes se encuentra en total acuerdo con el servicio, mientras que el $11 \%$ de los clientes manifiesta su indiferencia o desacuerdo con esta variable del estudio. En relación con el ambiente, el $42.1 \%$ de los clientes se encuentra satisfecho y, solamente, el $10 \%$, insatisfecho. Finalmente, el estudio concluyó que existe una relación significativa entre calidad de servicio y grado de satisfacción del cliente.

La investigación de Lescano (2017) se planteó como objetivo general determinar la calidad de servicio y la relación de esta con la satisfacción del cliente en la microempresa Comercial Lescano, Distrito Comas. El estudio trabajó con una población constituida por 200 clientes y una muestra de 132. Los resultados del estudio determinaron la presencia de una relación significativa entre la calidad de servicio con la satisfacción del cliente.

Al igual que otros autores, Larrea (1991) define la calidad de servicio como "la percepción que tiene un cliente acerca de la correspondencia entre el desempeño y las expectativas, relacionados con el conjunto de elementos secundarios, cuantitativos y cualitativos, de un producto o servicio principal" (p. 81). Mientras tanto, el autor Tarí Guilló (2000) señala que "la calidad del producto o servicio se convierte en objetivo fundamental de la empresa; ... en el enfoque moderno la perspectiva se amplía, considerando que va a ser toda la empresa la que va a permitir alcanzar esta meta" (p. 21-22).

Por otro lado, el mismo Larrea (1991) recuerda que, hace ya unos años, Paul Flipo señalaba como factores determinantes de la percepción de un buen servicio los diez siguientes elementos: fiabilidad (hacer las cosas bien a la primera), capacidad de respuesta, tangibilidad, 
cortesía, competencia, seguridad, credibilidad, accesibilidad, comunicación, comprensión y conocimiento del cliente. De igual modo, Larrea (1991) refiere que autores como Parasuraman, Zeithaml y Berry redujeron estos criterios a cinco: fiabilidad; capacidad de respuesta; tangibilidad; - aseguramiento (incluye cortesía, competencia, seguridad y credibilidad) y empatía (incluye accesibilidad, comunicación y conocimiento del cliente). Dado el crecimiento constante del rubro, la globalización y la competitividad, así como el incremento en la exigencia de la calidad de los servicios. el negocio de los restaurantes presenta ciertas dificultades, que se ponen de manifiesto en la insatisfacción de los clientes a la hora de evaluar los servicios de estos establecimientos. Pocas veces salen satisfechos.

Con el objetivo de aportar en la solución de estas dificultades, la presente investigación se propuso desarrollar nuevas estrategias de calidad del servicio que garanticen la adecuada atención al cliente en los restaurantes de la ciudad de Sullana.

El problema de estudio se definió a partir de la siguiente pregunta: ¿Cuál es la relación que existe entre la calidad del servicio y la satisfacción del cliente en los restaurantes Sullana? En definitiva, el objetivo general de la investigación se propuso determinar la relación entre la calidad del servicio y la satisfacción del cliente en los restaurantes señalados.

Entre los objetivos específicos, se pueden señalar los siguientes:

- Determinar la relación entre la tangibilidad y la satisfacción del cliente.

- Determinar la relación entre la capacidad de respuesta y la satisfacción del cliente.

- Determinar la relación entre la seguridad y la satisfacción del cliente.

Este trabajo se propone contribuir con nuevos conocimientos acerca de las variables de investigación en este tipo de estudios. Asimismo, los resultados de este documento podrán utilizarse como un referente base para otros investigadores que deseen realizar estudios similares sobre distintas empresas de la provincia e, incluso, de otros espacios geográficos de nuestro país.

Además, este estudio permitirá conocer mejor la realidad de las MYPE en nuestro medio, permitiendo así la formulación de nuevas teorías que incrementen la capacidad de estas para tomar decisiones y convertirlas en organizaciones más competitivas.

Todo esto favorecerá a la "comunidad empresarial" y emprendedora, que podrá valorar los beneficios que se obtienen de brindar una adecuada calidad de servicio a los clientes.

La investigación se fundamentó en diferentes conceptos y teorías de la calidad del servicio con el fin siempre de generar nuevas tendencias en el manejo de las variables implicadas en este tipo de procesos.

Cabe mencionar que en la actualidad la calidad del servicio y la satisfacción del cliente son variables que las empresas deben tener en cuenta para su constante fortalecimiento, siempre con el objetivo de llegar a obtener alto nivel de competitividad dentro de las MYPE. En este punto radica sobre todo la utilidad de esta investigación para la sociedad.

En definitiva, los resultados de esta investigación aportarán nuevos contenidos respecto a las variables implicadas en la calidad del servicio, acrecentando los conocimientos acerca del tema para posteriores investigaciones. 


\section{Diseño de la Investigación}

\section{Metodología}

El diseño de la presente investigación fue no experimental y el tipo de investigación, cuantitativa de nivel descriptivo-correlacional.

\section{Hipótesis}

Hipótesis general

Ho. La calidad del servicio no se relaciona significativamente con la satisfacción del cliente en los restaurantes.

Hi. La calidad del servicio se relaciona significativamente con la satisfacción del cliente en los restaurantes.

\section{Hipótesis específicas}

H1o. La tangibilidad no se relaciona significativamente con la satisfacción del cliente en los restaurantes de Sullana.

H1i. La tangibilidad se relaciona significativamente con la satisfacción del cliente en los restaurantes de Sullana.

H2o La capacidad de respuesta no se relaciona significativamente con la satisfacción del cliente en los restaurantes de Sullana.

H2i. La capacidad de respuesta se relaciona significativamente con la satisfacción del cliente en los restaurantes de Sullana.

H3o La seguridad no se relaciona significativamente con la satisfacción del cliente en los restaurantes de Sullana.

H3i. La seguridad se relaciona significativamente con la satisfacción del cliente en los restaurantes de Sullana.

\section{Población y Muestra}

La población estuvo conformada por los clientes de 20 MYPE del distrito de Sullana; por lo tanto, la población que se estudió es considerada infinita.

La muestra estuvo conformada por 384 clientes que acudieron a las MYPE del rubro restaurante del distrito de Sullana durante el año 2019. Para determinar la muestra, se aplicó la fórmula de muestreo para poblaciones infinitas.

\section{Instrumento} Likert.

Como instrumento se aplicó un cuestionario de 22 preguntas medidas con la escala de

\section{Plan de Análisis}

La base de datos de los encuestados se generó en Microsoft Excel 2016 y, para su tabulación, se utilizó como soporte el programa estadístico SPSS vs 25.0. También, se empleó la prueba de correlación de RHO SPEARMAN para probar las hipótesis planteadas, lo que permitió obtener aprobación y significancia. 


\section{Consideraciones Éticas}

Para el desarrollo de la investigación se respetaron los siguientes principios éticos:

Integridad científica: bajo este principio "el investigador debe proceder con rigor científico, asegurando la validez de sus métodos, fuentes y datos. Además, debe garantizar la veracidad en todo el proceso de investigación, desde la formulación, desarrollo, análisis, y comunicación de los resultados" (Uladech, 2021, p. 4).

Principio de libre participación y derecho a estar informado: en cumplimiento de este principio, se contó con el consentimiento previo, libre e informado de los sujetos investigados o titular de los datos [...] para los fines específicos establecidos en el proyecto (Uladech, 2021, p. 3).

\section{Resultados}

Mediante la información obtenida sobre la calidad del servicio de los restaurantes, se observa que la mayoría de los clientes encuestados $(68.23 \%)$ considera estar de acuerdo con la calidad del servicio, mientras que el $31.25 \%$ muestra ser indiferentes con respecto a esta variable. Por otro lado, un mínimo de clientes, el $0.52 \%$, está en desacuerdo. En conclusión, la mayor parte de los clientes manifiesta estar de acuerdo con la calidad del servicio ofrecida por los restaurantes (Tabla 1).

Tabla 1

Calidad del servicio

\begin{tabular}{|c|l|c|c|c|c|}
\hline \multicolumn{2}{|c|}{} & Frecuencia & \% & \% válido & $\%$ acumulado \\
\hline \multirow{3}{*}{ Válido } & $\begin{array}{l}\text { En } \\
\text { desacuerdo }\end{array}$ & 2 & 0,5 & 0,5 & 0.5 \\
\cline { 2 - 6 } & Indiferente & 120 & 31,3 & 31,3 & 31,8 \\
\cline { 2 - 6 } & $\begin{array}{l}\text { De } \\
\text { acuerdo }\end{array}$ & 262 & 68,2 & 68,2 & 100,0 \\
\cline { 2 - 6 } & Total & 384 & 100,0 & 100,0 & \\
\hline
\end{tabular}

Mediante la encuesta aplicada, se determina que el $69 \%$ de los encuestados está totalmente de acuerdo con la variable satisfacción del cliente. Asimismo, el $30.5 \%$ está de acuerdo con esta variable $\mathrm{Y}$ solo a un $0.5 \%$ le resulta indiferente este criterio. En conclusión, la mayoría de los clientes está satisfecho con los servicios que brindan los restaurantes (Tabla 2).

\section{Tabla 2}

Satisfacción del cliente

\begin{tabular}{|c|c|c|c|c|c|}
\hline \multicolumn{2}{|c|}{} & Frecuencia & $\%$ & $\%$ válido & \% acumulado \\
\hline \multirow{4}{*}{ Válido } & Indiferente & 2 & 0,5 & 0,5 & 0,5 \\
\cline { 2 - 6 } & De acuerdo & 117 & 30,5 & 30,5 & 31,0 \\
\cline { 2 - 6 } & $\begin{array}{l}\text { Totalmente } \\
\text { de acuerdo }\end{array}$ & 265 & 69,0 & 69,0 & 100,0 \\
\cline { 2 - 6 } & Total & 384 & 100,0 & 100,0 & \\
\hline
\end{tabular}


Por medio de la información obtenida sobre la calidad de servicio mediante la tangibilidad, se observa que la mayor cantidad de los encuestados $(45.8 \%)$ considera estar de acuerdo con este criterio, mientras que el $26.8 \%$ se manifiesta totalmente de acuerdo. Por otro lado, solo un $2.6 \%$ se encuentra en desacuerdo. En conclusión, la mayoría de encuestados considera estar de acuerdo con el servicio tangible, es decir, con sus instalaciones físicas, materiales atractivos y equipos modernos (Tabla 3 ).

Tabla 3

Tangibilidad

\begin{tabular}{|l|l|c|c|c|c|}
\hline \multicolumn{2}{|c|}{} & Frecuencia & $\%$ & \% válido & \% acumulado \\
\hline \multirow{7}{*}{ Válido } & $\begin{array}{l}\text { En } \\
\text { desacuerdo }\end{array}$ & 10 & 2,6 & 2,6 & 2,6 \\
\cline { 2 - 6 } & Indiferente & 95 & 24,7 & 24,7 & 27,3 \\
\cline { 2 - 6 } & De acuerdo & 176 & 45,8 & 45,8 & 73,2 \\
\cline { 2 - 6 } & $\begin{array}{l}\text { Totalmente } \\
\text { de acuerdo }\end{array}$ & 103 & 26,8 & 26,8 & 100,0 \\
\cline { 2 - 6 } & Total & 384 & 100,0 & 100,0 & \\
\hline
\end{tabular}

Mediante los resultados obtenidos con respecto al servicio de capacidad de respuesta de los restaurantes, se pudo identificar que la mayor parte de los clientes $(41.1 \%)$ está totalmente de acuerdo. El $39.1 \%$ está de acuerdo y solo el $1.6 \%$ se muestra en desacuerdo. En conclusión, los clientes manifiestan estar totalmente de acuerdo y de acuerdo con el servicio de capacidad de respuesta (Tabla 4).

\section{Tabla 4}

Capacidad de respuesta

\begin{tabular}{|c|l|c|c|c|c|}
\hline \multicolumn{2}{|c|}{} & Frecuencia & \% & \% válido & \% acumulado \\
\hline \multirow{7}{*}{ Válido } & $\begin{array}{l}\text { En } \\
\text { desacuerdo }\end{array}$ & 6 & 1,6 & 1,6 & 1,6 \\
\cline { 2 - 6 } & Indiferente & 70 & 18,2 & 18,2 & 19,8 \\
\cline { 2 - 6 } & De acuerdo & 150 & 39,1 & 39,1 & 58,9 \\
\cline { 2 - 6 } & $\begin{array}{l}\text { Totalmente } \\
\text { de acuerdo }\end{array}$ & 158 & 41,1 & 41,1 & 100,0 \\
\cline { 2 - 6 } & Total & 384 & 100,0 & 100,0 & \\
\hline
\end{tabular}

Por otro lado, el $57.8 \%$ de los clientes encuestados está de acuerdo con el servicio de seguridad, el $21.4 \%$ se encuentra totalmente de acuerdo y un porcentaje mínimo (1.04 \%) está totalmente en desacuerdo con el servicio de seguridad. En conclusión, los clientes manifiestan estar conformes con el servicio de seguridad gracias, sobre todo, a la seguridad de las transacciones y la confianza transmitida por los trabajadores, de quienes se resaltó su amabilidad, así como la capacidad y el conocimiento para resolver las dudas del cliente (Tabla $5)$. 
Tabla 5

Seguridad

\begin{tabular}{|c|l|c|c|c|c|}
\hline \multicolumn{2}{|c|}{} & Frecuencia & \% & \% válido & \% acumulado \\
\hline \multirow{7}{*}{ Válido } & $\begin{array}{l}\text { Totalmente } \\
\text { en } \\
\text { desacuerdo }\end{array}$ & 4 & 1,0 & 1,0 & 1,0 \\
\cline { 2 - 6 } & $\begin{array}{l}\text { En } \\
\text { desacuerdo }\end{array}$ & 12 & 3,1 & 3,1 & 4,2 \\
\cline { 2 - 6 } & Indiferente & 64 & 16,7 & 16,7 & 20,8 \\
\cline { 2 - 6 } & De acuerdo & 222 & 57,8 & 57,8 & 78,6 \\
\cline { 2 - 6 } & $\begin{array}{l}\text { Totalmente } \\
\text { de acuerdo }\end{array}$ & 82 & 21,4 & 21,4 & 100,0 \\
\cline { 2 - 6 } & Total & 384 & 100,0 & 100,0 & \\
\hline
\end{tabular}

\section{Prueba de hipótesis}

De acuerdo con el baremo de estimación de correlación de Spearman y considerando que el coeficiente RHO Spearman es 0.885 (88 \%), se puede afirmar que existe una correlación positiva alta. Además, el nivel de significancia es menor que 0.05 . Por tanto, se puede concluir que la calidad del servicio se relaciona significativamente con la satisfacción del cliente, lo que permite aceptar la hipótesis general, que indica la relación entre las variables calidad del servicio y satisfacción del cliente (Tabla 6).

Tabla 6

Correlaciones - Prueba de Hipótesis General

\begin{tabular}{|c|c|c|c|c|}
\hline & & & $\begin{array}{c}\text { Calidad del } \\
\text { Servicio } \\
\text { (Agrupada) }\end{array}$ & $\begin{array}{c}\text { Satisfacción } \\
\text { del cliente } \\
\text { (Agrupada) }\end{array}$ \\
\hline \multirow{6}{*}{$\begin{array}{l}\text { Rho de } \\
\text { Spearman }\end{array}$} & \multirow{3}{*}{$\begin{array}{l}\text { Calidad del } \\
\text { Servicio } \\
\text { (Agrupada) }\end{array}$} & $\begin{array}{l}\text { Coeficiente de } \\
\text { correlación }\end{array}$ & 1,000 & $0,885^{* *}$ \\
\hline & & Sig. (bilateral) & . & 0,000 \\
\hline & & $\mathbf{N}$ & 384 & 384 \\
\hline & \multirow{3}{*}{$\begin{array}{l}\text { Satisfacción } \\
\text { del cliente } \\
\text { (Agrupada) }\end{array}$} & $\begin{array}{l}\text { Coeficiente de } \\
\text { correlación }\end{array}$ & $0,885^{* *}$ & 1,000 \\
\hline & & Sig. (bilateral) & 0,000 & . \\
\hline & & $\mathbf{N}$ & 384 & 384 \\
\hline
\end{tabular}

**. La correlación es significativa en el nivel 0,01 (bilateral).

De acuerdo con el baremo de estimación de correlación de Spearman y considerando que el coeficiente RHO Spearman es $0.500(50 \%)$, se puede concluir que existe una correlación positiva moderada. Además, el nivel de significancia es menor que 0.01. De tal forma, se puede concluir que la tangibilidad se relaciona significativamente con la satisfacción del cliente, por lo cual H1i es la hipótesis aceptada (Tabla 7). 
Tabla 7

Correlaciones - Prueba de Hipótesis Especifica 1

\begin{tabular}{|c|c|c|c|c|}
\hline & & & $\begin{array}{l}\text { Tangibilidad } \\
\text { (Agrupado) }\end{array}$ & $\begin{array}{l}\text { Satisfacción } \\
\text { del cliente } \\
\text { (Agrupada) }\end{array}$ \\
\hline \multirow{6}{*}{$\begin{array}{l}\text { Rho de } \\
\text { Spearman }\end{array}$} & \multirow{3}{*}{$\begin{array}{l}\text { Tangibilidad } \\
\text { (Agrupada) }\end{array}$} & $\begin{array}{l}\text { Coeficiente de } \\
\text { correlación }\end{array}$ & 1,000 & $0,500^{* *}$ \\
\hline & & Sig. (bilateral) & . & 0,000 \\
\hline & & $\mathbf{N}$ & 384 & 384 \\
\hline & \multirow{3}{*}{$\begin{array}{l}\text { Satisfacción } \\
\text { del cliente } \\
\text { (Agrupada) }\end{array}$} & $\begin{array}{l}\text { Coeficiente de } \\
\text { correlación }\end{array}$ & $0,500^{* *}$ & 1,000 \\
\hline & & Sig. (bilateral) & 0,000 & . \\
\hline & & $\mathbf{N}$ & 384 & 384 \\
\hline
\end{tabular}

**. La correlación es significativa en el nivel 0,01 (bilateral).

De acuerdo con el baremo de estimación de correlación de Spearman y considerando que el coeficiente RHO Spearman es 0.613 , se puede afirmar que existe una correlación positiva moderada. Además, el nivel de significancia es menor que 0.01. Por tanto, se puede concluir que la capacidad de respuesta se relaciona significativamente con la satisfacción del cliente, por lo cual H2i es la hipótesis aceptada (Tabla 8).

\section{Tabla 8}

Correlaciones - Prueba de Hipótesis Específica 2

\begin{tabular}{|c|c|c|c|c|}
\hline & & & $\begin{array}{c}\text { Capacidad de } \\
\text { respuesta } \\
\text { (Agrupada) }\end{array}$ & $\begin{array}{c}\text { Satisfacción } \\
\text { del cliente } \\
\text { (Agrupada) } \\
\end{array}$ \\
\hline \multirow{6}{*}{$\begin{array}{l}\text { Rho de } \\
\text { Spearman }\end{array}$} & \multirow{3}{*}{$\begin{array}{l}\text { Capacidad de } \\
\text { respuesta } \\
\text { (Agrupada) }\end{array}$} & $\begin{array}{l}\text { Coeficiente de } \\
\text { correlación }\end{array}$ & 1,000 & $0,613^{* *}$ \\
\hline & & Sig. (bilateral) & . & 0,000 \\
\hline & & $\mathbf{N}$ & 384 & 384 \\
\hline & \multirow{3}{*}{$\begin{array}{l}\text { Satisfacción } \\
\text { del cliente } \\
\text { (Agrupada) }\end{array}$} & $\begin{array}{l}\text { Coeficiente de } \\
\text { correlación }\end{array}$ & $0 ., 613^{* *}$ & 1,000 \\
\hline & & Sig. (bilateral) & 0,000 & . \\
\hline & & $\mathbf{N}$ & 384 & 384 \\
\hline
\end{tabular}

**. La correlación es significativa en el nivel 0,01 (bilateral).

De acuerdo con el baremo de estimación de correlación de Spearman y considerando que el coeficiente RHO Spearman es 0.456 (45\%), se obtiene que existe una correlación positiva alta. Además, el nivel de significancia es menor que 0.05 . Por tanto, se puede concluir que la seguridad del servicio se relaciona significativamente con la satisfacción del cliente, por lo cual H3i es la hipótesis aceptada (Tabla 9). 
Tabla 9

Correlaciones - Prueba de Hipótesis específica 3

\begin{tabular}{|c|c|c|c|c|}
\hline & & & $\begin{array}{l}\text { Seguridad } \\
\text { (Agrupada) }\end{array}$ & $\begin{array}{c}\text { Satisfacción } \\
\text { del cliente } \\
\text { (Agrupada) }\end{array}$ \\
\hline \multirow{6}{*}{$\begin{array}{l}\text { Rho de } \\
\text { Spearman }\end{array}$} & \multirow{3}{*}{$\begin{array}{l}\text { Seguridad } \\
\text { (Agrupada) }\end{array}$} & $\begin{array}{l}\text { Coeficiente de } \\
\text { correlación }\end{array}$ & 1,000 & $0,456^{* *}$ \\
\hline & & Sig. (bilateral) & . & 0,000 \\
\hline & & $\mathbf{N}$ & 384 & 384 \\
\hline & \multirow{3}{*}{$\begin{array}{l}\text { Satisfacción } \\
\text { del cliente } \\
\text { (Agrupada) }\end{array}$} & $\begin{array}{l}\text { Coeficiente de } \\
\text { correlación }\end{array}$ & $0,456^{* *}$ & 1,000 \\
\hline & & Sig. (bilateral) & 0,000 & . \\
\hline & & $\mathbf{N}$ & 384 & 384 \\
\hline
\end{tabular}

**. La correlación es significativa en el nivel 0,01 (bilateral).

\section{Discusión}

A partir de los resultados obtenidos en esta investigación, se puede asegurar que existe una relación entre la tangibilidad y la satisfacción del cliente, es decir que los clientes se encuentran satisfechos con las instalaciones físicas, materiales atractivos y equipos modernos (ver Tabla 3). Este resultado es similar al encontrado por Pintado Alberca (2018), quien identificó que "son buenos los servicios brindados ya que emplean adecuadamente los elementos tangibles como materiales de comunicación para ofertar sus productos a los clientes".

Además, se determinó que existe relación entre la capacidad de respuesta y la satisfacción del cliente (ver Tabla 4), es decir que los clientes se encuentran totalmente satisfechos con el servicio de capacidad de respuesta, dado que los empleados comunican el tiempo de espera del servicio demandado, lo realizan con rapidez y muestran buena disposición para ayudar a los clientes. Este resultado es similar a los de C. Coronel, (2016), quien concluyó que la mayoría de encuestados considera que el tiempo de espera para ser atendido es adecuado. También se constató que los clientes están conformes con el servicio de seguridad brindado, debido, sobre todo, a la confianza transmitida por los empleados, la amabilidad, la capacidad de resolver dudas de los clientes y la seguridad brindada en las transacciones (ver Tabla 5). Este resultado es similar al encontrado por C. Coronel (2016), quien concluyó que los clientes están satisfechos con la atención recibida, ya que los empleados del restaurante estudiado son amables.

Por otro lado, se validó la hipótesis general al quedar demostrada la correlación existente entre la calidad de servicio y la satisfacción del cliente en los restaurantes de Sullana, puesto que el nivel de significancia es 0,000 (ver Tabla 6). Este resultado es similar al de López (2018), quien en su estudio estableció que existe correlación significativa entre la calidad de servicio y la satisfacción al cliente, dado que la significancia fue 0,000 .

Luego de procesar los datos recabados, se concluyó que la correlación entre la tangibilidad y la satisfacción al cliente es positiva moderada (ver Tabla 7). Este resultado es similar al de Lescano (2017), quien determinó que existe relación entre la tangibilidad y la satisfacción al cliente. 
Como resultado se obtuvo que, de acuerdo con el baremo de estimación de correlación de Spearman, existe una correlación positiva moderada entre la capacidad de respuesta y la satisfacción del cliente (ver Tabla 8). Este resultado contrasta con el encontrado por Lescano (2017), quien concluyó que existe correlación positiva moderada entre la capacidad de respuesta y la satisfacción del cliente.

Finalmente, la tercera hipótesis específicas consistió en determinar si la seguridad se relaciona significativamente con la satisfacción del cliente en los restaurantes de Sullana. Luego de procesar los datos, se determinó que existe una correlación positiva alta (ver Tabla 9). Cabe indicar que este resultado es igual al encontrado por Lescano (2017), quien concluyó que la seguridad y la satisfacción del cliente presentan una correlación positiva alta.

\section{Conclusiones}

La mayoría de los clientes está de acuerdo con la calidad del servicio de los restaurantes en Sullana durante el periodo 2019.

La mayoría de los clientes están totalmente satisfechos con la calidad del servicio de los restaurantes en Sullana durante el periodo 2019.

La mayoría de los clientes está de acuerdo con la calidad del servicio en cuanto la tangibilidad, la capacidad de respuesta y la seguridad de los restaurantes de Sullana durante el periodo 2019.

La calidad del servicio y la tangibilidad, la capacidad de respuesta y la seguridad se relacionan significativamente con la satisfacción del cliente en los restaurantes de Sullana durante el periodo 2019.

Se recomienda a los empresarios de los restaurantes de Sullana mantener el nivel de calidad del servicio en los establecimientos a través de constantes capacitaciones a sus colaboradores en torno a la atención al cliente y las competencias de cada área de trabajo; además, se deben realizar siempre estudios de mercado que determinen cuáles son las necesidades reales de los clientes y cuáles son sus expectativas; se debe mantener la satisfacción de los clientes implementando políticas de atención al cliente, cumpliendo con lo ofrecido en el menor tiempo posible y brindando una atención personalizada; se debe invertir constantemente en la infraestructura y el equipamiento moderno, lo que permite mejorar los tiempos de atención al cliente.

\section{Reconocimientos}

Este trabajo ha sido financiado parcialmente por la Universidad Católica Los Ángeles de Chimbote.

El autor desea expresar su agradecimiento a los empresarios encuestados por su participación en la investigación.

\section{Referencias}

Coronel, C. (2016). Calidad de servicio y grado de satisfacción del cliente en el restaurant Pizza Hut en el centro comercial Mega Plaza Lima; 2016. [Tesis de Grado, Universidad Señor de Sipán].

http://repositorio.uss.edu.pe//handle/20.500.12802/2707 
Coronel, J., Basantes, R., \& Vinueza, A. (2019). Un estudio de la calidad del servicio en restaurantes de mariscos (Ecuador). Espacios, 40(7), 9-19.

Gastromakers, Cultura Gastronómica. (9 de julio de 2018). Clasificación de los restaurantes. https://www.gastromakers.com/2018/07/09/clasificacion-de-los-restaurantes-2/

Instituto Nacional de Estadística e Informática (INEI), (2018, junio 22). Negocios de restaurantes aumentó 3,04 $\%$ abril de 2018 y creció por décimo tercer mes consecutivo.

https://www.inei.gob.pe/prensa/noticias/negocios-de-restaurantes-aumento-304-abril-de-2018-y-creciopor-decimo-tercer-mes-consecutivo-10812/

Jiménez, C. (2016). Análisis de los Procesos de Producion y Servicio al Cliente del Restaurante Pizza al Passo, en Ricaurte Cundinamarca [Tesis de Grado, Universidad de Cundinamarca].

https://docplayer.es/73956330-Analisis-de-los-procesos-de-producion-y-servicio-al-cliente-delrestaurante-pizza-al-passo-en-ricaurte-cundinamarca-2016.html

Larrea, P. (1991). Calidad de servicio: Del marketing a la estrategia. Ediciones Díaz de Santos. https://elibro.net/es/ereader/uladech/52840?page $=78$.

Lescano, M. (2017). Calidad de servicio y su relación con la satisfacción del cliente en la Microempresa Comercial Lescano, Distrito Comas, AÑO 2017 [Tesis de Grado, Universidad César Vallejo]. https://repositorio.ucv.edu.pe/handle/20.500.12692/16610

López, D. (2018). Calidad del servicio y la satisfacción de los clientes del Restaurante Rachy's de la ciudad de Guayaquil. [Tesis de Grado, Universidad Católica de Santiago de Guayaquil]. http://repositorio.ucsg.edu.ec/handle/3317/9867

Pintado Alberca, D. (2018). Caracterización de la calidad del servicio al cliente y rentabilidad en las Mypes en el sector servicio, rubro restaurantes del distrito de Pampas de Hospital - Tumbes, 2017. [Tesis de Grado, Universidad Católica los Ángeles de Chimbote]. http://repositorio.uladech.edu.pe/handle/123456789/3942

Rodríguez, G., \& Venturo, S. (2007). Ampay Perú 357 listas para entender cómo somos los peruanos. Aguilar.

Tarí Guilló, J. J. (2000). Calidad total: Fuente de ventaja competitiva. Publicaciones Universidad de Alicante. https://biblioteca.org.ar/libros/133000.pdf

Vera, J., \& Trujillo, A. (2017). Escala mexicana de calidad en el servicio en restaurantes (EMCASER). Innovar, 27(63), 43-60. https://doi.org/10.15446/innovar.v26n63.60665 